SUBJECT AREAS:

POLYMERS

MECHANICAL PROPERTIES

Received

24 March 2014

Accepted

27 June 2014

Published

16 July 2014

Correspondence and requests for materials should be addressed to C.H.L. (chlu@tju.edu.

\section{Polydimethylsiloxane Microspheres via Wet Surface Chemical Oxidation}

\author{
Jian Yin' ${ }^{1}$ Xue Han', Yanping $\mathrm{Cao}^{2} \&$ Conghua Lu'
}

${ }^{1}$ School of Materials Science and Engineering, Tianjin University, Tianjin 300072, P. R. China, ${ }^{2}$ AML, Department of Engineering Mechanics, Tsinghua University, Beijing 100084, P. R. China.

Here we introduce a simple low-cost yet robust method to realize spontaneously wrinkled morphologies on spherical surfaces. It is based on surface chemical oxidation of aqueous-phase-synthesized polydimethylsiloxane (PDMS) microspheres in the mixed $\mathrm{H}_{2} \mathrm{SO}_{4} / \mathrm{HNO}_{3} / \mathrm{H}_{2} \mathrm{O}$ solution. Consequently, curvature and overstress-sensitive wrinkles including dimples and labyrinth patterns are successfully induced on the resulting oxidized PDMS microspheres. A power-law dependence of the wrinkling wavelength on the microsphere radius exists. The effects of experimental parameters on these tunable spherical wrinkles have been systematically investigated, when the microspheres are pre-deposited on a substrate. These parameters include the radius and modulus of microspheres, the mixed acid solution composition, the oxidation duration, and the water washing post-treatment. Meanwhile, the complicated chemical oxidation process has also been well studied by in-situ optical observation via the microsphere system, which represents an intractable issue in a planar system. Furthermore, we realize surface wrinkled topographies on the whole microspheres at a large scale, when microspheres are directly dispersed in the mixed acid solution for surface oxidation. These results indicate that the introduced wet surface chemical oxidation has the great potential to apply to other complicated curved surfaces for large-scale generation of well-defined wrinkling patterns, which endow the solids with desired physical properties.

s one type of surface instabilities, surface wrinkling has attracted increasing interest due to its incomparable unique applications for non-lithographic patterning of a surface over areas of interest ${ }^{1-4}$, characterization of film properties ${ }^{5}$, photo/electric devices with high flexibilities and enhanced performances ${ }^{6-8}$, microstructured templates of the related fields ${ }^{9-11}$, and so on. It is well accepted that wrinkling always occurs on a film/substrate system to minimize the system potential energy, when the compressive stress $\sigma$ is higher than a critical value $\sigma_{c}^{1,2,12,13}$. This compressive stress $\sigma$ is induced during the in-plane compression as a consequence of the film/substrate strain mismatch. In a plane strain compression, the wavelength $\lambda$ of the resulting undulated wrinkles and the critical stress $\sigma_{c}$ are determined by $\lambda=2 \pi h\left(\frac{\overline{E_{f}}}{3 \overline{E_{s}}}\right)^{1 / 3}$ and $\sigma_{c}=\frac{\overline{E_{f}}}{4}\left(\frac{3 \overline{E_{s}}}{\overline{E_{f}}}\right)^{2 / 3}$, respectively ${ }^{1,2,12,13}$. Here $h$ is the film thickness, $\bar{E}$ is the plane strain modulus given by $\frac{E}{1-v^{2}}, E$ is the Young's modulus, and $v$ is the Poisson's ratio. Subscripts of $f$ and $s$ refer to the film and substrate, respectively.

In the past decade, much progress has been made in revelation and application of the surface wrinkling physics in planar film/substrate systems ${ }^{14-21}$. Indeed, many natural and industrial wrinkling phenomena often happen on non-planar film/substrate systems, e.g., the familiar aging skin and some dried fruits. Furthermore, the geometrical constraint engenders apparently different wrinkling behaviors on a curved surface from those on a planar surface ${ }^{13,22-27}$. For example, in a spherical $\mathrm{Ag}$ core $/ \mathrm{SiO}_{2}$ shell system, Cao and Yin et al. utilized the finite element method (FEM) to investigate the correlation of the wrinkling patterns with the normalized substrate radius of curvature $R / h_{R}$ and the overstress $\sigma^{R} / \sigma_{c}^{R^{22}}$. Here $\sigma^{R}$ and $\sigma_{c}^{R}$ refer to the applied stress and the critical wrinkling stress on a spherical core/shell system with the shell thickness $h_{R}$ and the substrate radius $R$, respectively. It is found that smaller $R / h_{R}$ and $\sigma^{R} / \sigma_{c}^{R}$ result in triangular dentlike wrinkling patterns on the spherical surface. Whereas, higher $R / h_{R}$ and $\sigma^{R} / \sigma_{c}^{R}$ tend to elicit typically labyrinth topographies. Meanwhile, the FEM result 
reveals the following scaling relation between the wrinkling wavelength $\lambda^{R}$ and $R / h_{R}^{22}$

$$
\frac{\lambda^{R}}{R}=a\left(\frac{R}{h_{R}}\right)^{b}
$$

where $a$ and $b$ are two constants. Eq. 1 is verified by Trindade et al., in their study surface wrinkles were generated in such a way that elastomeric polyurethane/polybutadiene (PU/PBDO) composite spheres were exposed to UV irradiation, followed by solvent swelling ${ }^{27}$. This curvature-sensitive surface wrinkling is further studied by other researchers. Thereinto, Crosby et al. explored the surface wrinkling in the supported spherical caps of poly(dimethyl siloxane) (PDMS)

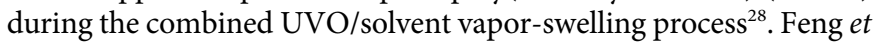
al. investigated the surface wrinkling and wrinkling morphology transition on a core-shell soft sphere using the volumetric growth theory of finite deformation as well as the experimental observation of a gradually dehydrated green peas ${ }^{25}$. The influences of the substrate curvature and the applied overstress generated by differential growth/shrinkage on the wrinkling topologies have been explored ${ }^{25,26,28}$. In the case of a bigger $\Omega$ or a smaller $\sigma^{R} / \sigma_{c}^{R}$ (or $\bar{g}$ ) applied, ordered dimples or buckyball-like wrinkling patterns appear on the spherical surface. In the case of a lower $\Omega$ or a bigger $\sigma^{R} / \sigma_{c}^{R}$ (or $\bar{g})$, the wrinkling patterns are the energetically-preferred labyrinth morphologies. Here $\Omega$ and $\bar{g}$ are the introduced dimensionless curvature parameter ${ }^{28}$ and the growth/shrinking factor ${ }^{25}$, respectively. $\Omega$ is defined $\mathrm{as}^{29}$ :

$$
\Omega=2 \sqrt{\left(1-v_{f}^{2}\right)}\left(h_{R} / R\right)\left(\overline{E_{f}} / 3 \overline{E_{s}}\right)^{2 / 3}
$$

Thus $\sigma_{c}^{R}$ is determined by ${ }^{29}$ :

$$
\frac{\sigma_{c}^{R}}{\sigma_{c}} \approx 1+\Omega^{2}
$$

From Eqs. 2, 3, it is seen that $\sigma_{c}^{R}$ is dependent on $R / h_{R}$ and $\overline{E_{f}} / \overline{E_{s}}$.

So far, considerable theoretical and experimental efforts have been made towards the above intriguing spherical wrinkling. However only several technical routes have been proposed to create wrinkling patterns on spherical surfaces successfully ${ }^{13,22-28}$. What's more, a number of limitations have been recognized in these methods. For instance, in the as-reported spherical core/shell systems, the Ag core/ $\mathrm{SiO}_{2}$ shell spheres were prepared by thermal co-evaporation of the mixed precursors at high temperature ${ }^{22}$. With respect to the $\mathrm{PU} /$ PBDO Janus spher $\mathrm{e}^{27}$ and the replicated PDMS spherical cap supported by a flat PDMS slab ${ }^{28}$, only hemispherical surface was involved in the wrinkling. Furthermore, the methods are not suitable for a large-scale fabrication on curved surfaces. Recently, surface wrinkles on polymer microspheres have been obtained based on one-pot emulsion polymerization of cross-linked polystyrene (PS) microspheres ${ }^{30}$, or microfluidic formation of PS emulsion droplets ${ }^{31}$ or self organized precipitation of liquid-crystalline microparticles ${ }^{32}$ followed by controlled solvent evaporation, which are not desirable for non-spherical curved surfaces yet. Besides, the correlation between the wrinkling patterns on the curved substrate with the mechanical parameters of the film/substrate bilayer has not been explored using systematic experiments. Therefore, it is of great importance to develop a simple low-cost approach for a large-scale fabrication of wrinkled patterns on curved systems from a scientific and technical point of view.

In this paper, we develop a surface chemical oxidation of a mixed acid solution for the large-scale wrinkling on PDMS microspheres with typically non-planar surfaces. The dependence of the curvature/ overstress-sensitive wrinkling behaviors on the oxidation processing parameters including the radius and modulus of microsphere has been investigated systematically. It is noted that although this wet surface oxidation processing has been utilized by Shih's ${ }^{33}$ and Watanabe' $s^{34}$ groups on a planar PDMS substrate for creating surface wrinkles, this study represents the first effort to apply the method to a curved micro-sized system. Furthermore, the detailed information regarding the complicated surface oxidation has been revealed by insitu optical microscope observation, which appears to be impossible to achieve in a planar system. Compared with the previous studies $^{22-28,30-32}$, our system and the method developed here have the following advantages. Surface oxidation processing provides a simple, rapid, scalable, high-output method to generate the stiff oxidized shell on the microspheres and simultaneously trigger surface wrinkles on the spherical surface. No special preparation equipment and surface geometries, and no external wrinkling stimuli (e.g., stretching/compressing, solvent swelling, and heating) are required in the above acid oxidation processing, indicative of the enormous potential to apply it for wrinkling on arbitrarily non-planar surfaces on a large scale.

\section{Results}

Figure 1a shows the SEM image of the aqueous-phase-synthesized $\operatorname{PDMS}_{(10: 1)}$ microparticles from a 10:1 weight ratio of the base/ curing agent. It is seen that these solid PDMS ${ }_{(10: 1)}$ microparticles have typically spherical morphologies with smooth surfaces. Indeed, synthesis of high quality of PDMS microspheres is the pre-requisite for our investigation of the wrinkling on spherical surface. In the previous reports ${ }^{35-37}$, microsphere aggregations and surface defects (e.g., the concave or porous feature) are always the challenging issues in the solution-phase synthesis of PDMS particles. In our case, these obvious morphological improvements are obtained due to the different synthesis procedure (see the details in the Methods). For instance, the initial degassing decreases the introduction of air bubble. More importantly, high concentration of poly(vinyl alcohol) as an effective capping/stabilizing agent coupled with two sequential curing stages (i.e., firstly at a lower temperature and then at a higher temperature) promotes the stability of the PDMS precursor-based emulsion droplet, and thus leads to the final smooth isolated PDMS microspheres. Certainly, compared to the PDMS microbeads synthesized by the expensive microfluidic approach ${ }^{36,37}$, the polydispersity of our PDMS microspheres is still relatively large. However, a wide radius distribution provides a good platform for simultaneous investigation of the influence of the microsphere size on the spherical wrinkling behaviors. Similarly, $\operatorname{PDMS}_{(\mathrm{n}: 1)}$ microspheres with other weight ratios of the base/curing agent (e.g., $\mathrm{n}: 1=5: 1,15: 1$, and $20: 1$ ) have also been successfully prepared (SI: Fig. S1a-c).

In the following surface oxidation processing, two procedures (i.e., $\mathrm{P}_{\mathrm{I}}$ and $\mathrm{P}_{\mathrm{II}}$ ) are explored. For $\mathrm{P}_{\mathrm{I}}$, a glass slide with PDMS microspheres coated on its surface was immersed into the mixed strong acid solution of $\mathrm{H}_{2} \mathrm{SO}_{4} / \mathrm{HNO}_{3} / \mathrm{H}_{2} \mathrm{O}$ at the reaction temperature of $T_{\mathrm{r}}$. After the oxidation time of $t_{\mathrm{r}}$, it was taken out promptly and immersed immediately into a certain $\mathrm{pH}$ aqueous solution with the temperature of $T_{\mathrm{w}}$ for the washing duration of $t_{\mathrm{w}}$. Finally, the as-prepared oxidized sample was dried at $70^{\circ} \mathrm{C}$. For $\mathrm{P}_{\mathrm{II}}$, the microspheres were directly dispersed in the mixed acid solution for the above surface oxidation and the subsequent water washing. Unless otherwise noted, the following experiment is performed via the $\mathrm{P}_{\mathrm{I}}$ procedure.

Firstly, the $P_{I}$ procedure is utilized to investigate the spherical wrinkling behaviors via the wet surface chemical oxidation. Under standard conditions (i.e., $\mathrm{PDMS}_{(10: 1)}$ microsphere, the mixed $\mathrm{H}_{2} \mathrm{SO}_{4} / \mathrm{HNO}_{3} / \mathrm{H}_{2} \mathrm{O}$ solution with the volume ratio of $66: 11: 12$, surface oxidation at $T_{\mathrm{r}}=35^{\circ} \mathrm{C}$ for $t_{\mathrm{r}}=1 \mathrm{~min}$, washing by a $\mathrm{pH}=$ 7 aqueous solution at $T_{\mathrm{w}}=70^{\circ} \mathrm{C}$ for $t_{\mathrm{w}}=30 \mathrm{~min}$ ), the resultant morphologies of the oxidized $\mathrm{PDMS}_{(10: 1)}$ microspheres were recorded by SEM (Fig. 1b). Compared to the initial smooth surface shown in Fig. 1a, the oxidized PDMS microspheres have rough microtextured surfaces. The zoomed SEM images clearly dem- 

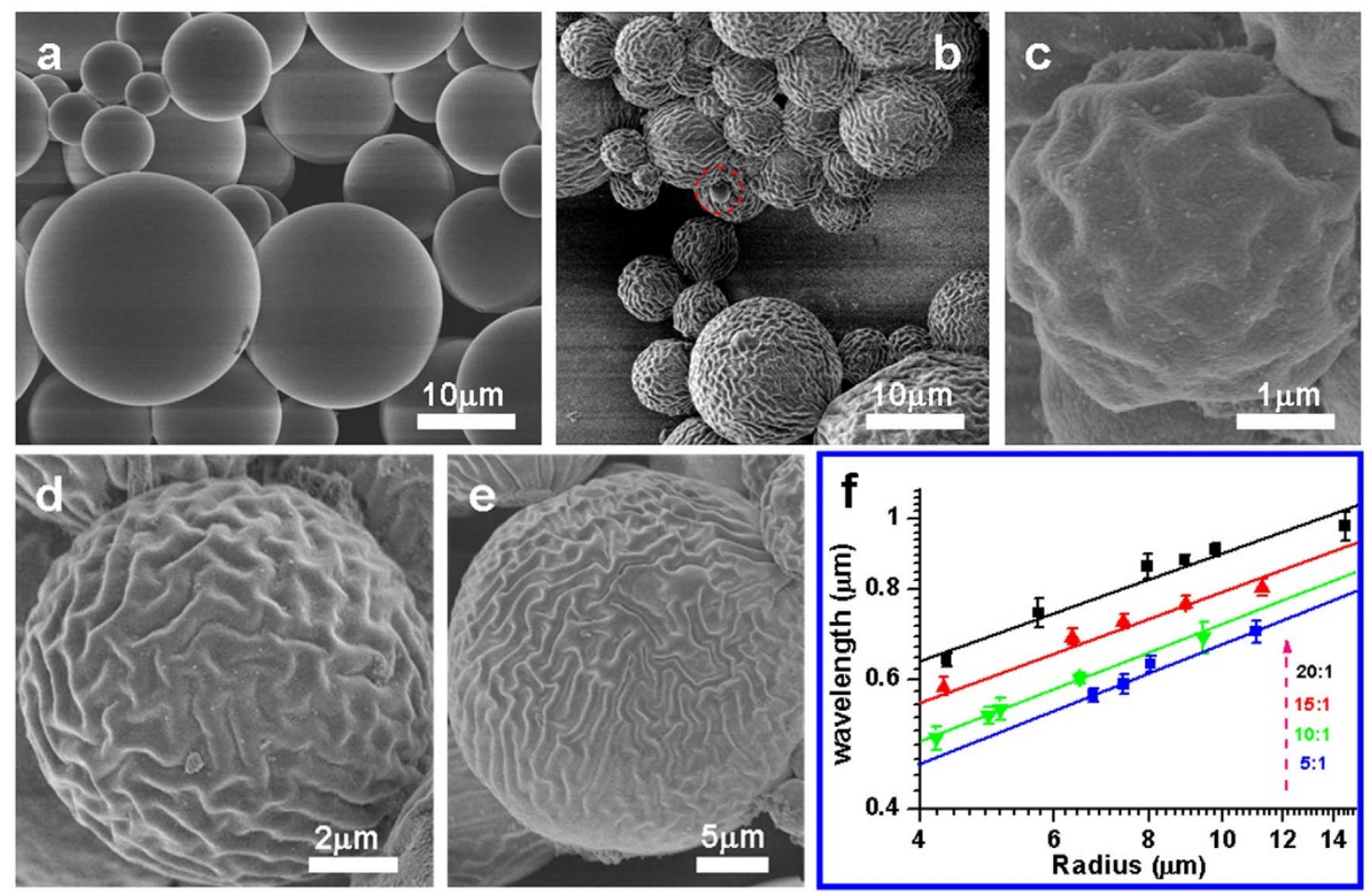

Figure 1 SEM images of PDMS $_{(10: 1)}$ microspheres before (a) and after (b-e) the $\mathrm{P}_{\mathrm{I}}$ processing. Zoomed PDMS $(10: \mathbf{1})$ microspheres with the radius R: $\left.\mathrm{c}\right)$ $\sim 2.25 \mu \mathrm{m}$; d) $\sim 4.75 \mu \mathrm{m}$; e) $\sim 15 \mu \mathrm{m}$. Frame f shows the $\lambda^{R} \sim \mathrm{R}$ relationship for different $E_{P D M S_{(n: 1)}}$.

onstrate that these surface textures belong to typical wrinkle morphologies (Fig. 1c-e). Furthermore, when the radius $R$ of PDMS $_{(10: 1)}$ microspheres increases from $\sim 2.25 \mu \mathrm{m}$ to $\sim 4.75 \mu \mathrm{m}$, the corresponding wrinkles change from the dimple (or buckyballlike) (Fig. 1c) to labyrinth patterns (Fig. 1d). A careful examination further shows that under the same conditions, no surface wrinkling is induced on a small radius of $\mathrm{PDMS}_{(10: 1)}$ microsphere (circled one in Fig. 1b), owing to the requirement of a larger critical wrinkling stress according to Eqs. $2,3^{28,29}$.

The influence of the Young's modulus of $\mathrm{PDMS}_{(\mathrm{n}: 1)}$ microspheres $\left(E_{P D M S_{(n: 1)}}\right)$ on the wrinkling patterns is given in Fig. S1d-f (SI). In the current case, $E_{P D M S_{(n: 1)}}$ is well tuned by the weight ratio (n:1) of the PDMS base/curing agent. Furthermore, the $E_{P D M S_{(n: 1)}}$ of $\mathrm{PDMS}_{(\mathrm{n}: 1)}$ microsphere can be considered equal to that of the corresponding planar PDMS $\mathrm{Pn}_{(\mathrm{n})}$ film. According to the planar data reported in Ref. $38, E_{P D M S_{(n: 1)}}$ is estimated to be $\sim 2.6 \mathrm{MPa}, 1.8 \mathrm{MPa}, 0.9 \mathrm{MPa}$, and $0.5 \mathrm{MPa}$, when $\mathrm{n}: 1=5: 1,10: 1,15: 1$, and $20: 1$, respectively. Evidently, the wrinkling behavior on these spherical surfaces (SI: Fig. S1d-f) is similar to that on the $\operatorname{PDMS}_{(10: 1)}$ microspheres (Fig. 1b-e). When compared the roughly same radius sizes of $\operatorname{PDMS}_{(\mathrm{n}: 1)}$ microspheres (Fig. 1b and SI: Fig. S1e,f), we see the bridged dimples are favorable on stiffer PDMS microspheres (e.g., SI: Fig. S1d). It should be originated from the increase of $\sigma_{c}^{R}$ for more rigid spherical substrates and the decrease of the resultant overstress.

Figure if shows the relation of the wrinkling wavelength $\lambda^{R}$ with the radius $R$ and the modulus $E_{P D M S_{(n: 1)}}$ of $\mathrm{PDMS}_{(\mathrm{n}: 1)}$ microspheres, respectively. Here the wrinkling wavelength $\lambda^{R}$ is mainly estimated on the base of the recorded SEM images. It is found that the power law behavior between $\lambda^{R}$ and $R$ exists for the same $E_{P D M S_{(n: 1)}}$ applied (Fig. 1f). Additionally, consistent with the theoretical predictions given by Eqs.2,3, the $\lambda^{R} \sim R$ plots for different moduli of $\mathrm{PDMS}_{(\mathrm{n}: 1)}$ microspheres are approximately parallel to each other in the double logarithmic coordinate (Fig. 1f). Meanwhile, the wrinkles triggered on stiffer PDMS microspheres have a smaller wrinkle wavelength under the same conditions.
It is known that when exposed to the mixed strong acid solution, the surface oxidation reaction occurs on PDMS microspheres with the formation of an oxidized $\mathrm{SiO}_{\mathrm{x}}$ layer, just as the case of the planar PDMS substrate applied ${ }^{33,34}$. Consequently, a film/substrate spherical system composed of the $\mathrm{SiO}_{\mathrm{x}}$ outerlayer and the underlying PDMS microsphere is generated. Under the standard oxidation conditions, the thickness $h_{R}$ of the oxidized layer can be assumed to be equal for different radii of $\operatorname{PDMS}_{(10: 1)}$ microspheres. Thus in Fig. 1b-e, the dimple patterns are formed in the oxidized $\operatorname{PDMS}_{(10: 1)}$ microspheres with a larger $h_{R} / R$ (i.e., $\Omega$ ), whereas the labyrinth patterns take over in the presence of a smaller $h_{R} / R$. These substrate curvature-dependent wrinkle morphologies are in good agreement with the previous experimental and theoretical results $s^{22,25,26,28}$. As for the power law between the wrinkling wavelength $\lambda^{R}$ and the sphere radius $R$ (Fig. 1f) is also in accord with the previous results ${ }^{22-24,27}$. For example, according to the numerical result of Yin et al., the critical wrinkling wavelength $\lambda_{c r}^{R}$ in a cylindrical substrate is given by ${ }^{23,24}$ :

$$
\lambda_{c r}^{R}=2 \pi h_{R}\left(\frac{R}{h_{R}}\right)^{\frac{1}{4}}\left(\frac{\bar{E}_{f}}{12 \bar{E}_{s}}\right)^{\frac{1}{4}} .
$$

It can be simplified as:

$$
\lg \lambda_{c r}^{R}=\frac{1}{4} \lg R+C
$$

In the work of Cao et al., the critical wrinkling wavelength on $\mathrm{Ag}$ core $/ \mathrm{SiO}_{2}$ shell composite microspheres is shown in Eq.1 with the constants of $a=3.0$ and $b=-0.8$, respectively ${ }^{22}$. Alternatively, Eq.1 can be written as

$$
\lg \lambda_{c r}^{R}=0.2 \lg R+C^{\prime}
$$

From the experimental result of Trindade et al. on the hemispherical surface wrinkling in elastomeric PU/PBDO spheres, we have $\mathrm{e}^{27}$

$$
\lg \lambda^{R}=0.18 \lg R+C^{\prime \prime}
$$


In the above equations, $C, C^{\prime}$, and $C^{\prime \prime}$ are the functions of the shell thickness $h_{R}$ and the modulus mismatch of $\overline{E_{f}} / \overline{E_{s}}$, respectively. In our case (i.e., $h_{R}$ and $\overline{E_{f}} / \overline{E_{s}}$ remain unchanged when the oxidation reaction time, the mixed acid composition, and microsphere modulus are fixed), $\lambda^{R}$ and $R$ approximately satisfy the following linear relation:

$$
\lg \lambda^{R}=0.3 \lg R-0.33 \text {. }
$$

Apparently, the slope in Eq. (8) is somehow larger than those shown in Eqs.5-7. This difference may be attributed to the different wrinkling systems and experimental conditions $\mathrm{s}^{22,27}$. Additionally, this deviation from the theoretical predictions might as well be due to the fact that the oxidation procedure introduces a cross-linkage gradient rather than a layer with a constant elastic property, as it is usually assumed in the theoretical work.

Here we study the effect of the mixed acid composition on the wrinkling morphologies on $\operatorname{PDMS}_{(10: 1)}$ microspheres (Fig. 2). Different from the as-reported composition modulation of the mixed acid solution via the heating-induced volatilization of $\mathrm{HNO}_{3}{ }^{34}$, the current case is achieved by simply mixing the corresponding volume ratio of acids and water directly. Firstly, the change of $\mathrm{HNO}_{3}$ content is studied (Fig. 1b-e and Fig. 2a-g). For example, when $V_{\mathrm{H}_{2} \mathrm{SO}_{4}}: V_{\mathrm{HNO}_{3}}: V_{\mathrm{H}_{2} \mathrm{O}}$ is set to be $66: \mathrm{x}: 12$ (x: $\mathrm{HNO}_{3}$ content), the mixed acid solution with a too high (e.g., $x=40$, Fig. $2 a$ ) or too low $x$ (e.g., $x=5$, Fig. $2 g$ ) can not engender the wrinkling on the spherical surface. The relatively suitable $\mathrm{HNO}_{3}$ content for the wrinkling is found to be $15 \sim 7$ with $V_{\mathrm{H}_{2} \mathrm{SO}_{4}}: V_{\mathrm{HNO}_{3}}: V_{\mathrm{H}_{2} \mathrm{O}}$ of 66: $(15 \sim 7): 12$ (Fig. 1b-e and Fig. $2 \mathrm{c}-\mathrm{f}$ ). It is known that the oxidation reactivity of the mixed acid solution decreases as the $\mathrm{HNO}_{3}$ content decreases ${ }^{34}$. Thus a small $\mathrm{HNO}_{3}$ content with a low oxidation reactivity can not elicit the formation of the rigid oxidized outerlayer in the PDMS substrate because PDMS has a good surface chemical stability (Fig. 2g). When the oxidation reactivity is too high, the oxidized PDMS microspheres deform severely and adhere to each other (Fig. 2a). Meanwhile, a thicker and stiffer oxidized outerlayer is generated, resulting in a higher $\sigma_{c}^{R}$ expected from Eqs.2,329. However the swelling-induced $\sigma^{R}$ from the surface oxidation processing, which will be discussed later, is still lower than $\sigma_{c}^{R}$. Consequently no wrinkling happens in this case (Fig. 2a). As for the same large size of the PDMS microspheres (e.g., $\sim 5 \mu \mathrm{m}$ in the radius, the circled one in Fig. 2f), a careful examination shows that the wrinkling morphology evolves from the labyrinth patterns to the dimples, when the volume ratio of the strong acid mixtures varies from 66:(15 11):12 (Fig. $1 \mathrm{~b}$ and Fig. 2 c,d) to 66:(9 7):12 (Fig. 2e,f). In the latter case, a low overstress is obviously at least one pre-requisite for the formation of dimples on spherical surfaces. Additionally, these results are reminiscent of the fine modulation of $\sigma_{c}^{R}$ and $\sigma^{R}$ through alteration of the mixed acid composition, and the internal relation of the spherical wrinkling patterns with $\sigma^{R} / \sigma_{c}^{R}$ and $h_{R} / R$. According to Eqs.2, $3^{28,29}$, it is known that no wrinkling is induced on too small microspheres due to the sharp increase in $\sigma_{c}^{R}$. However, when the mixed acid solution with the volume ratio of 66:( $(9 \sim 7): 12$ is used, no wrinkling happens on big PDMS microspheres yet (denoted by red arrows in Fig. 2e.f). It is assumed to be the induced $\sigma^{R}$ lower than $\sigma_{c}^{R}$. From the latter result shown in Fig. S2 (SI), we know that bigger microspheres have a smaller swelling ratio in comparison with smaller ones under the same conditions.

Similarly, $\mathrm{H}_{2} \mathrm{SO}_{4}$ composition in the mixed acid solution is also important. A lower $\mathrm{H}_{2} \mathrm{SO}_{4}$ content can not lead to surface wrinkling on PDMS microspheres (Fig. 2h,i). This is due to the fact that the oxidation ability of the mixed acid solution comes from the synergetic effect of $\mathrm{HNO}_{3}$ and $\mathrm{H}_{2} \mathrm{SO}_{4}{ }^{33}$. In addition, the concentration of the mixed acid solution also needs to be carefully considered (Fig. 2j, k). The mixed acid solution with a high concentration (e.g., $V_{\mathrm{H}_{2} \mathrm{SO}_{4}}: V_{\mathrm{HNO}_{3}}=66: 11$, no water added) leads to a porous film with full disappearance of the original PDMS microspheres due to the excessively strong oxidation etching (Fig. 2j). As for a relatively low concentration (Fig. 2k), low surface oxidation reactivity is not enough to oxidize PDMS microspheres, just as the case of a low content of $\mathrm{HNO}_{3}$ (Fig. 2g) or $\mathrm{H}_{2} \mathrm{SO}_{4}$ (Fig. 2h,i) applied.

In addition, the relation of the oxidation time $t_{\mathrm{r}}$ with the wrinkling behavior has also been explored (Fig. 3). From the recorded SEM images (Fig. 3a-e), it is seen that $t_{\mathrm{r}}$ has no obvious effect on the wrinkling morphology. A shorter oxidation time $t_{\mathrm{r}}$ (e.g., $30 \mathrm{~s}$, Fig. 3a), even $10 \mathrm{~s}$ (data not shown here), roughly results in the
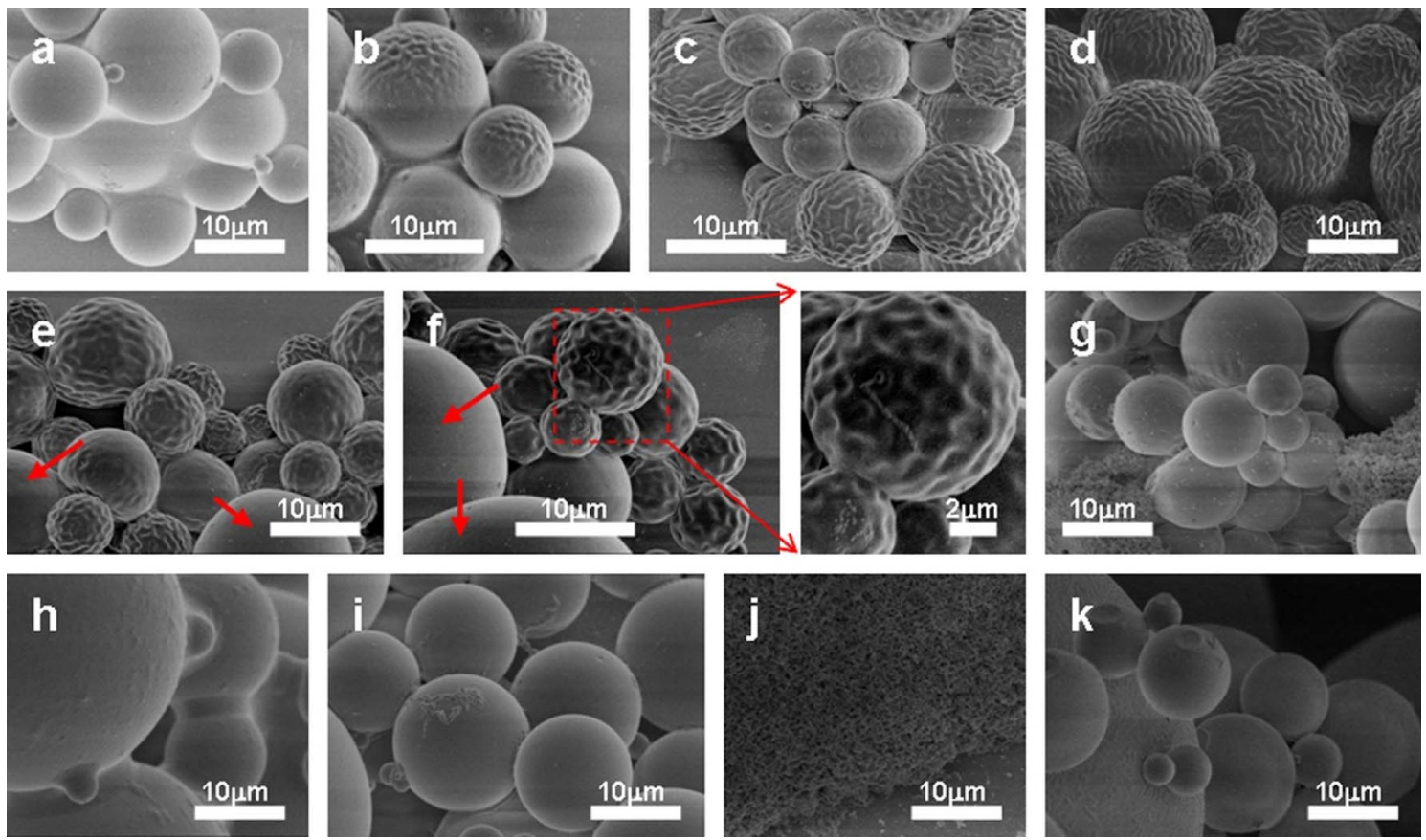

Figure $2 \mid$ SEM images of the $\mathrm{P}_{\mathrm{I}}$-processed PDMS $(10: 1)$ microspheres with different $\left.\left.V_{\mathrm{H}_{2} \mathrm{SO}_{4}}: V_{\mathrm{HNO}_{3}}: V_{\mathrm{H}_{2} \mathrm{O}}: \mathrm{a}\right) 66: 40: 12 ; \mathrm{b}\right) 66: 20: 12$; c) $66: 15: 12$; d) $66: 13: 12$; e) $66: 9: 12$;f) $66: 7: 12$; g) $66: 5: 12$; h) $30: 11: 12$; i) $10: 11: 12$; j) $66: 11: 0$; k) $66: 11: 24$. 

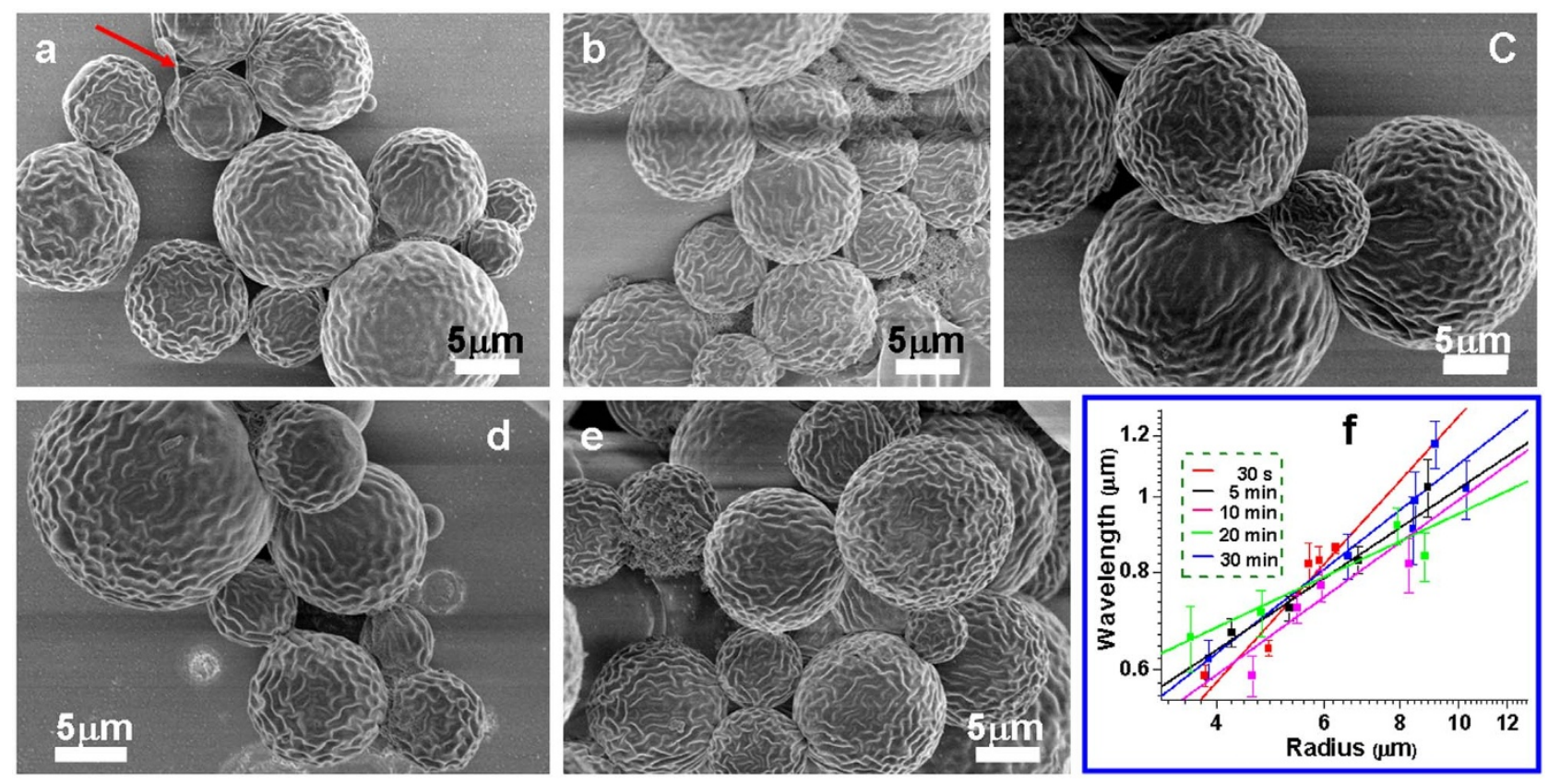

Figure $3 \mid$ SEM images of the $P_{I^{-}}$processed PDMS $(10: 1)$ microspheres with different $t_{\mathrm{r}}$ : a) $30 \mathrm{~s}$, b) 5 min; c) 10 min; d) 20 min; e) 30 min. Frame f shows the relation between $\lambda^{R}$ and $\mathrm{R}$ in the double logarithmic coordinate.

similar wrinkling patterns with those from a longer oxidation time $t_{\mathrm{r}}$ (e.g., $30 \mathrm{~min}$, Fig. 3e). Namely, the typically labyrinth and dimple wrinkles are elicited on PDMS microspheres with a small and large $h_{R} / R$ (Fig. 1b-e and Fig. 3a-e), respectively, regardless of the oxidation duration $t_{\mathrm{r}}$. Meanwhile a linear correlation between the microsphere radius $R$ and the resulting wrinkle wavelength $\lambda^{R}$ still retains in the double logarithmic coordinate. Unexpectedly, for the same size of PDMS $(10: 1)$ microspheres, the wrinkle wavelength $\lambda^{R}$ has no obvious dependence on the oxidation time $t_{\mathrm{r}}$ under the currently selected conditions (Fig. 3f). This unforeseen result implies that the thickness $h_{R}$ of the as-formed $\mathrm{SiO}_{\mathrm{x}}$ layer is approximately the same for all the as-investigated $t_{\mathrm{r}}$, which will be discussed in detail later.

In the above $\mathrm{P}_{\mathrm{I}}$, PDMS microspheres were deposited on a glass slide before the surface oxidation reaction. It is convenient for the in situ optical observation of the surface morpholological evolution to reveal the complicated oxidation process and the corresponding spherical wrinkling behaviors, which will be performed subsequently. The oxidized PDMS microspheres can also be promptly separated from the mixed strong acid solution, especially in the case of a very short oxidation time applied. Evidently, the $P_{I}$ procedure is not suitable for a scalable process. Furthermore, there is no wrinkling on the acid-untreated region of the microspheres that is in contact with the deposited substrate or other microspheres (denoted by the red arrow in Fig. 3a).

Bearing these issues in mind, we further develop the $\mathrm{P}_{\text {II }}$ procedure. Just as described in Methods and schemed in Fig. 4a, a large number of PDMS microspheres were transferred to a sintered glass filter (Fig. 4a (1)). Under continuous stirring, the mixed acid solution was carefully added (Fig. 4a (2)). After a certain duration of the surface oxidation reaction, the mixed acid solution was pumped out, and then a large amount of washing aqueous solution was immediately added to clean the oxidized PDMS microspheres thoroughly (Fig. 4a (3). During the above oxidation reaction and water washing, the continuous stirring was employed to prevent the contact of PDMS microspheres with other surfaces. As expected, a large-scale production of PDMS microspheres with wrinkled patterns on the whole spherical surfaces has been successfully realized via $P_{\text {II }}$ (Fig. 4b,c). Additionally, no obvious difference in the wrinkling behavior has been observed between $\mathrm{P}_{\mathrm{I}}$ and $\mathrm{P}_{\mathrm{II}}$.

\section{Discussion}

Although the oxidation-induced morphological change has been well documented $33,34,39$, the systematical experimental observation and the detailed information regarding the complicated surface oxidation reaction are inaccessible to a planar PDMS system. Furthermore, the involved surface oxidation-induced wrinkling mechanism is not clear yet. Here the PDMS microsphere system provides the excellent chance to study it. For the convenience of the in-situ optical observation of the whole $\mathrm{P}_{\mathrm{I}}$ processing, the PDMS microspheres were pre-deposited on the glass slide.

Firstly we investigate the diameter change in the dry state as a function of the oxidation time $t_{\mathrm{r}}$ and the $\mathrm{PDMS}_{(\mathrm{n}: 1)}$ modulus via the $\mathrm{P}_{\mathrm{I}}$ procedure (SI: Fig. S2). Later we carry out the in-situ optical observation to monitor the morphological evolution of the PDMS $_{(10: 1)}$ microspheres during the $\mathrm{P}_{\mathrm{I}}$ processing with different $t_{\mathrm{r}}$ applied (Fig. 5). Here $r_{D}$ is introduced to estimate the rate of the diameter change with $r_{D}=\frac{D_{t}-D_{0}}{D_{0}} \times 100 \%$, where $D_{0}$ and $D_{t}$ are the microsphere diameters before and after the processing for $t$, respectively. Thus the positive/negative $r_{D}$ means the microsphere diameter increase/decrease and the corresponding volume swelling/ shrinking. In order to exclude the influence of the diameter size on the resultant $r_{D}$, individual $\mathrm{PDMS}_{(10: 1)}$ microsphere with roughly the same diameter (e.g., $\sim 50 \mu \mathrm{m}$ ) is tracked for every selected oxidation duration $t_{\mathrm{r}}$. In Fig. $5, \mathrm{a}_{\mathrm{i}}, \mathrm{b}_{\mathrm{i}}, \mathrm{c}_{\mathrm{i}}$, and $\mathrm{d}_{\mathrm{i}}$ denote the initial state, the terminated acid oxidation, the water-washing post-treatment, and the dried state, respectively. Therefore $\left(a_{i} \rightarrow b_{i}\right),\left(b_{i} \rightarrow c_{i}\right)$ and $\left(c_{i} \rightarrow d_{i}\right)$ correspond to the three stages of the $P_{I}$ processing: mixed acid oxidation, washing post-treatment, and oven drying, respectively. It is found that the diameter/volume evolution with the $P_{I}$ processing is strongly dependent on the applied oxidation time $t_{\mathrm{r}}$ (Fig. 5).

Based on the results shown in Fig. S2 and Fig. 5, in combination with the previously-reported information, the surface oxidation process during the $P_{I}$ processing and the corresponding spherical wrinkling can be revealed explicitly. Namely, once immersed into the mixed $\mathrm{H}_{2} \mathrm{SO}_{4} / \mathrm{HNO}_{3} / \mathrm{H}_{2} \mathrm{O}$ solution, surface chemical oxidation takes place on PDMS microspheres. On the one hand, the oxidation fragment of $\mathrm{CH}_{3}-\mathrm{Si}-\mathrm{O}$ in the PDMS surface yields numerous silanol 

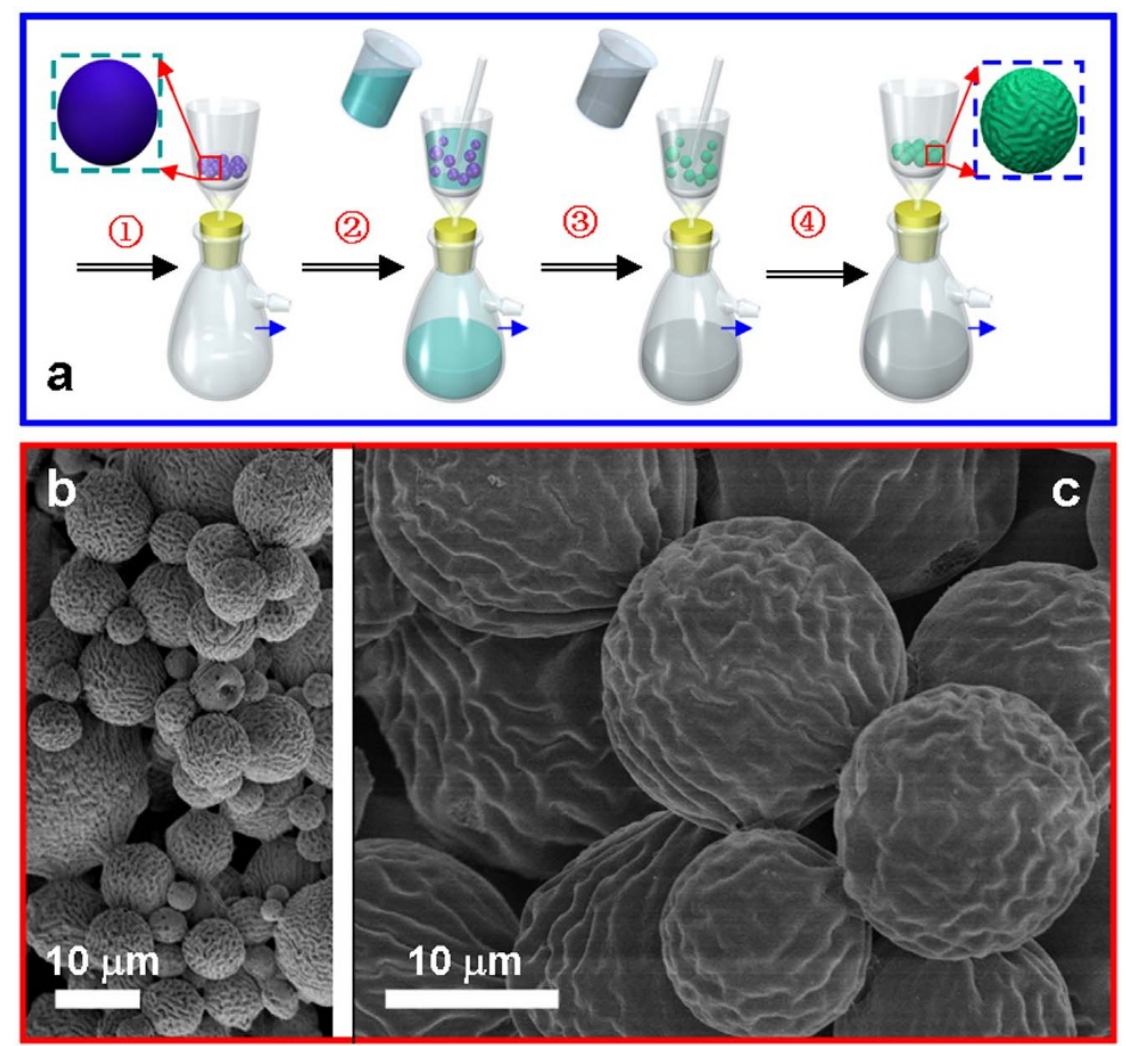

Figure $4 \mid$ (a) Schematic illustration of the large-scale fabrication via $\mathrm{P}_{\mathrm{II}}$ including four steps: (1) transfer of microspheres to a sintered glass filter; (2) mixed acid oxidation under continuous stirring; (3) thorough water washing; (4) pumping out/drying. (b,c) Typical SEM images of the resulting wrinkled microspheres.

groups, which further self-condensate to form -Si-O-Si-. These oxidation reactions have been verified in a planar PDMS substrate by infrared spectrometry and X-ray photoelectron spectroscopy ${ }^{33,34}$. Consequently, a thin rigid $\mathrm{SiO}_{\mathrm{x}}$ layer similar to silicon resin or silica is formed on the PDMS microsphere and an oxidized shell/pristine PDMS core microsphere is fabricated. On the other hand, soluble silicates and formaldehyde are also formed from acid oxidation cleavage of some methyl groups simultaneously ${ }^{33,34,39}$. This leads to the above diameter decrease and the volume shrinking for the oxidized microspheres (Fig. $5 a_{i} \rightarrow b_{i}$ and Fig. S2). Naturally, PDMS $(n: 1)$ microsphere with a high cross-linkage density and $E_{P D M S}$ has a relatively low volume shrinking ratio due to a better resistance to the acid oxidation (Fig. S2c). A longer oxidation time $t_{\mathrm{r}}$ leads to more serious oxidation etching with a final larger $r_{D}$ (Fig. $5 a_{i} \rightarrow b_{i}$ and Fig. $\mathrm{S} 2 \mathrm{a})$. However the as-formed $\mathrm{SiO}_{\mathrm{x}}$ layer not only can stand the further acid oxidation, but also acts as a barrier layer to protect the internal PDMS core from oxidation/etching. As a result, we observe during the mixed acid oxidation (Fig. $5 \mathrm{a}_{\mathrm{i}} \rightarrow \mathrm{b}_{\mathrm{i}}$ ), the morphological evolution with the initial fast decrease, the intermediate slow decrease, and the final saturation in the diameter size, although
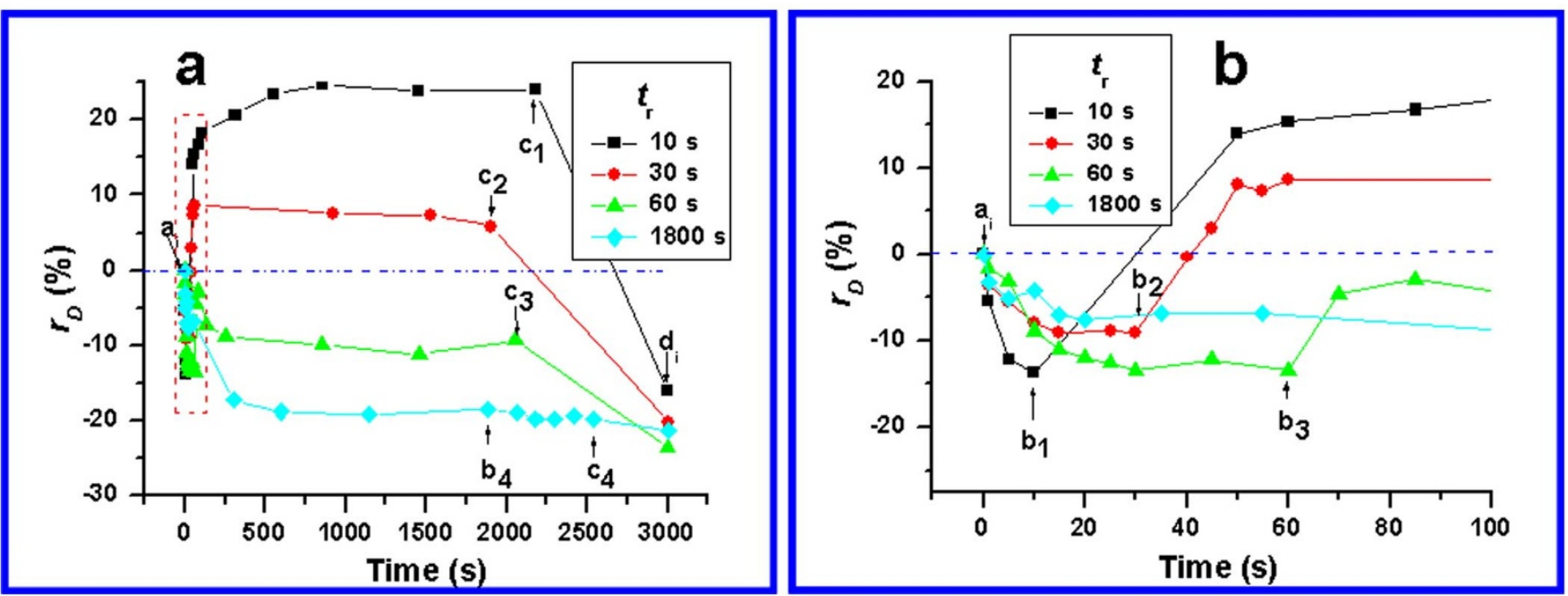

Figure $5 \mid$ a) Plot of the rate of the diameter change $\left(r_{D}\right)$ during the $\mathrm{P}_{\mathrm{I}}$ processing of PDMS $(10: 1)$ microspheres with different $\left.t_{\mathrm{r}}: \mathrm{a}_{\mathrm{i}} \rightarrow \mathrm{b}_{\mathrm{i}}\right)$ the mixed acid oxidation; $b_{i} \rightarrow c_{i}$ ) the water-washing post-treatment; $\left.c_{i} \rightarrow d_{i}\right) 70^{\circ} \mathrm{C}$ drying. b) The magnification of the circled part in a). 
self-condensation of the as-formed silanol groups into the insoluble $\mathrm{SiO}_{\mathrm{x}}$ layer and soluble silicates exists simultaneously. The above double functions of the as-formed $\mathrm{SiOx}$ layer are uncovered by the stability experiment of the $\mathrm{P}_{\mathrm{I}}$-processed PDMS microspheres, i.e., no obvious changes both in the diameter (data not shown) and the aswrinkled morphologies (SI: Fig. S3) occur during the second $\mathrm{P}_{\mathrm{I}}$ processing of the as-oxidized microspheres. These superior properties also endow the $\mathrm{P}_{\mathrm{I}}$-processed PDMS microspheres with one saturated thickness of the oxidized layer, which has been verified by the unexpected dependence of $\lambda^{R}$ on $t_{\mathrm{r}}$ shown in Fig. 3. This result is in sharp contrast with that of Watanabe et $a l^{34}$, who observed the beginning increase and the subsequent decrease in the oxidized layer thickness with the oxidation processing of the planar PDMS substrate.

During the acid oxidation, the as-formed silanol groups are hydrophilic. They can be swollen by the mixed acid solution and water. This swelling combined with the simultaneous self-condensation cross-linking fixation results in the surface wrinkling on the PDMS microspheres, once the swelling-induced compressive stress $\sigma^{R}$ is higher than $\sigma_{c}^{R}$. Certainly, the extent of the self-condensation of the hydrophilic silanol groups into the insoluble $\mathrm{SiO}_{\mathrm{X}}$ layer at the stage of the acid oxidation is chiefly determined by the acid oxidation time $t_{\mathrm{r}}$. For a short oxidation time $t_{\mathrm{r}}$ applied, a part of the silanol groups take part in the self-condensation. Furthermore, these silanol groups have no enough time to complete the thorough self-condensation into the insoluble $\mathrm{SiO}_{\mathrm{x}}$ layer with a high cross-linkage density. Consequently, the as-formed $\mathrm{SiO}_{\mathrm{x}}$ layer with the hydrophilic property, companied with the residual polar silanol groups, is swollen by the washing water, leading to the reverse volume increase at this washing stage (Fig. $5 b_{i} \rightarrow c_{i}$ ). The shorter the $t_{r}$ is, the higher the swelling degree (i.e., the more positive $r_{D}$ ) of the resultant PDMS microspheres will be (Fig. $5 \mathrm{~b}_{1} \rightarrow \mathrm{c}_{1}$ and $\mathrm{b}_{2} \rightarrow \mathrm{c}_{2}$ ). When $t_{\mathrm{r}}$ is long enough, the highly cross-linked $\mathrm{SiO}_{\mathrm{x}}$ layer formed at this oxidation stage can not be swollen again by the washing water (Fig. $5 b_{4} \rightarrow c_{4}$ ). Certainly these hydrophilic SiOx layer and the residual silanol groups can further react for the full self-condensation at the subsequent stages. This means the washing post-treatment will have an effect on the resultant wrinkling, just as shown in Fig. S4 (SI), which is also dependent on the applied $t_{\mathrm{r}}$.

Based on the above discussions, it can be concluded that the surface oxidation processing in the mixed acid solution has an effect not only on the swelling-induced compressive stress $\sigma^{R}$, but also on the critical wrinkling stress $\sigma_{c}^{R}$ because the latter is tightly related to the thickness $h_{R}$ and the Young's modulus $E$ of the rigid oxidized layer according to Eq. $3^{28,29}$. Roughly speaking, the swelling extent, the thickness, and the rigidity of the as-formed oxidized layer may increase with the oxidation reactivity of the mixed acid solution. Therefore a suitable composition of the mixed acid solution is required for the surface wrinkling on the PDMS microspheres. This has been validated by the effect of the mixed acid composition on the wrinkling behaviors shown in Fig. 2.

\section{Conclusion}

In summary, isolated solid PDMS microspheres synthesized by the simple aqueous phase are used as the compliant spherical substrate for surface wrinkling. The surface chemical oxidation in the mixed $\mathrm{H}_{2} \mathrm{SO}_{4} / \mathrm{HNO}_{3} / \mathrm{H}_{2} \mathrm{O}$ solution is introduced not only to form the oxidized rigid shell, but also to obtain the swelling-induced compressive stresses for generating spontaneous surface wrinkling on the PDMS microspheres. The resultant stable wrinkle morphologies exhibit the typically dimple and labyrinth patterns, which are sensitive to the substrate radius of curvature $R / h_{R}$ and the overstress $\sigma^{R} / \sigma_{c}^{R}$ applied. Here, $R / h_{R}$ and $\sigma^{R} / \sigma_{c}^{R}$ have been well tuned by the experimental conditions such as the radius and the modulus of the PDMS microspheres, the composition of the mixed acid solution, and the oxidation reaction duration. It is found that the wrinkling wavelength $\lambda^{R}$ and the microsphere radius $\mathrm{R}$ have the power-law dependency. Additionally, the complicated reaction process, which is inaccessible to the planar PDMS system, has been revealed explicitly by in-situ optical microscope observation. Owing to the excellent physical/chemical properties of PDMS, the as-prepared PDMS-based wrinkled microspheres with the fine surface textures are expected to apply in a variety of areas including wetting, friction, adhesion, gas sensors, functional coatings and micro/nano-fabrication ${ }^{32,36,37}$. Evidently, the combination of the substrate curvature with the imposed stress engineering and the mixed acid oxidation provides a simple versatile strategy to pattern arbitrarily curved inaccessible surfaces to other techniques on a large scale. Moreover, the method and the results reported here may be helpful in understanding the stress-driven morphogenesis of some natural curved biological systems from a mechanical point of view, although there complicated biological and chemical processes are involved ${ }^{13,22-26}$.

\section{Methods}

Materials. The base/curing agent (Sylgard 184) of polydimethylsiloxane (PDMS) precursor was purchased from Dow Corning Corporation. Poly(vinyl alcohol) (PVA, $M_{\mathrm{w}}=20000-30000,88+\%$ hydrolyzed) was purchased from J\&K Scientific Ltd. Concentrated $\mathrm{H}_{2} \mathrm{SO}_{4}$ (95-98 wt \%), concentrated $\mathrm{HNO}_{3}$ (65-68 wt \%) were purchased from National Pharmaceutical Group Corporation (Sinopharm, Beijing, China).

Synthesis of PDMS Microspheres. The PDMS base/curing agent at a designed weight ratio (e.g., $5: 1,10: 1,15: 1$, and $20: 1$ ) was mixed thoroughly, followed by degassing for $30 \mathrm{~min}$. Then $30 \mathrm{~mL}$ of $15 \mathrm{wt} \%$ PVA aqueous solution was added to $0.6 \mathrm{~g}$ of the above base/curing agent. The formed emulsion droplet was stirred at room temperature for $12 \mathrm{~h}$ and then at $80^{\circ} \mathrm{C}$ for another $12 \mathrm{~h}$ to complete the curing reaction. The as-prepared PDMS microspheres were centrifuged at $3000 \mathrm{rpm}$ and washed with water for several times. For simplicity, $\mathrm{PDMS}_{(\mathrm{n}: 1)}$ microspheres represent the cured microspheres from the $\mathrm{n}: 1$ weight ratio of the base/curing agent.

Preparation of the Mixed Acid Solution. The mixed strong acid solution with the volume ratio of $x: y: z$ was prepared by directly mixing $x \mathrm{~mL}$ of concentrated $\mathrm{H}_{2} \mathrm{SO}_{4}, y$ $\mathrm{mL}$ of concentrated $\mathrm{HNO}_{3}$ and $z \mathrm{~mL}$ of $\mathrm{H}_{2} \mathrm{O}$. Caution: the mixing and stirring should be extremely careful due to the strong oxidation reactivity.

Surface Wrinkling on PDMS $_{(\mathrm{n}: 1)}$ Microspheres via Surface Chemical Oxidation. Two typical procedures (i.e., $\mathrm{P}_{\mathrm{I}}$ and $\mathrm{P}_{\mathrm{II}}$ ) were utilized for the surface oxidationinduced wrinkling. For $\mathrm{P}_{\mathrm{I}}$, the $\mathrm{PDMS}_{(\mathrm{n}: 1)}$ microspheres were deposited on a clean glass slide firstly. Then the microsphere-coated substrate was carefully immersed into the above freshly-prepared $\mathrm{H}_{2} \mathrm{SO}_{4} / \mathrm{HNO}_{3} / \mathrm{H}_{2} \mathrm{O}$ solution at the reaction temperature of $T_{\mathrm{r}}$. After the oxidation time of $t_{\mathrm{r}}$, it was taken out promptly and immersed immediately into a certain $\mathrm{pH}$ aqueous solution with the temperature of $T_{\mathrm{w}}$ for the washing duration of $t_{\mathrm{w}}$. Finally, the oxidized sample was dried at $70^{\circ} \mathrm{C}$ for subsequent characterization. For $\mathrm{P}_{\mathrm{II}}$, the PDMS microspheres were transferred to a $\mathrm{G} 4$ sintered glass filter. Then the mixed acid solution at $T_{\mathrm{r}}$ was carefully added under continuous stirring with a glass rod. After a certain duration $\left(t_{\mathrm{r}}\right)$ of surface oxidation reaction, the mixed acid solution was pumped out and a large amount of the washing aqueous solution at $T_{\mathrm{w}}$ was added to clean the oxidized PDMS microspheres thoroughly. During the above oxidation reaction and water washing, the stirring was also employed. Similarly, the resultant PDMS microspheres were dried at $70^{\circ} \mathrm{C}$ for the subsequent characterization. Unless otherwise noted, the surface oxidation processing was carried out via the $\mathrm{P}_{\mathrm{I}}$ procedure. For example, the effect of the experimental parameters on the spherical wrinkling behaviors and the in-situ optical observation of the microsphere diameter change were performed via the $\mathrm{P}_{\mathrm{I}}$ processing. The standard conditions are as follows: the $\mathrm{PDMS}_{(10: 1)}$ microspheres were treated in the $\mathrm{H}_{2} \mathrm{SO}_{4} / \mathrm{HNO}_{3} / \mathrm{H}_{2} \mathrm{O}$ mixture $(66: 11: 12 \mathrm{vol} \%)$, as well $T_{\mathrm{r}}=35^{\circ} \mathrm{C}$, $T_{\mathrm{w}}=70^{\circ} \mathrm{C}, t_{\mathrm{r}}=1 \mathrm{~min}, t_{\mathrm{w}}=30 \mathrm{~min}$, and $\mathrm{pH}=7$, respectively.

Characterization. The as-prepared original PDMS microspheres and the mixed acid treated PDMS microspheres were characterized by a Hitachi S4800 field-emission scanning electron microscope (FE-SEM). In order to improve the sample conductivity for SEM characterization, Pt film was sputtered with an E-1045 ion sputter (HITACHI) at the pressure of $7 \mathrm{~Pa}$ and the current of $15 \mathrm{~mA}$. The in-situ optical microscope observation of the morphological evolution of the PDMS microspheres during the whole $\mathrm{P}_{\mathrm{I}}$ processing was carried out on an inverted Observer A1 microscope (Zeiss, Germany) equipped with a charge-coupled device camera. In order to realize the in-situ observation, the microsphere-coated glass slide was put in a transparent vessel. Then several drops of the mixed acid solution were added carefully on it. After the designed oxidation duration $t_{\mathrm{r}}$, abundant deionized water was carefully added to terminate the surface oxidation and to wash the as-oxidized microspheres. During the above two steps, the in-situ observation was carried out. As for the last step of heating drying in the $\mathrm{P}_{\mathrm{I}}$ procedure, the corresponding diameter 
change was monitored and the same PDMS microspheres can be well tracked again under optical microscope through the as-marked feature.

1. Bowden, N. et al. Spontaneous formation of ordered structures in thin films of metals supported on an elastomeric polymer. Nature 393, 146-149 (1998).

2. Genzer, J. \& Groenewold, J. Soft matter with hard skin: from skin wrinkles to templating and material characterization. Soft Matter 2, 310-323 (2006).

3. Schweikart, A. \& Fery, A. Controlled wrinkling as a novel method for the fabrication of atterned surfaces. Microchim. Acta 165, 249-263 (2009).

4. Mei, Y., Kiravittaya, S., Harazim, S. \& Schmidt, O. G. Principles and applications of micro and nanoscale wrinkles. Mater. Sci. Eng. R 70, 209-224 (2010).

5. Stafford, C. M. et al. Buckling-based metrology for measuring the elastic moduli of polymeric thin films. Nat. Mater. 3, 545-550 (2004).

6. Khang, D. Y., Rogers, J. A. \& Lee, H. H. Mechanical buckling: mechanics, metrology, and stretchable electronics. Adv. Funct. Mater. 19, 1526-1536 (2009).

7. Zhu, W. J. et al. Structure and electronic transport in graphene wrinkles. Nano Lett. 12, 3431-3436 (2012)

8. Kim, J. B. et al. Wrinkles and deep folds as photonic structures in photovoltaics. Nat. Photonics 6, 327-332 (2012).

9. Hyun, D. C. et al. Buckling- assisted patterning of multiple polymers. Adv. Mater. 22, 2642-2646 (2010).

10. Lee, S. G. et al. Evaporation-induced self-alignment and transfer of semiconductor nanowires by wrinkled elastomeric templates. Adv. Mater. 25, 2162-2166 (2013).

11. Cai, X. et al. Fabrication of ultrafine soft-matter arrays by selective contact thermochemical reaction. Sci. Rep. 3, 1780 (2013).

12. Huang, Z. Y., Hong, W. \& Suo, Z. Nonlinear analyses of wrinkles in a film bonded to a compliant substrate. J. Mech. Phys. Solids 53, 2101-2118 (2005).

13. Li, B., Cao, Y. P., Feng, X. Q. \& Gao, H. J. Mechanics of morphogical instabilities and surface wrinkling in soft materials: a review. Soft Matter 8, 5728-5745 (2012).

14. Yang, S., Khare, K. \& Lin, P. C. Harnessing surface wrinkle patterns in soft matter. Adv. Funct. Mater. 20, 2550-2564 (2010)

15. Singamaneni, S. \& Tsukruk, V. V. Buckling instabilities in periodic composite polymeric materials. Soft Matter 6, 5681-5692 (2010)

16. Ding, W. L. et al. Well-defined orthogonal surface wrinkles directed by wrinkled boundary. Soft Matter 9, 3720-3726 (2013)

17. Yang, Y. et al. Controlled free edge effects in surface wrinkling via combination of external straining and selective $\mathrm{O}_{2}$ plasma exposure. Langmuir 29, 7170-7177 (2013)

18. Yoo, P. J. \& Lee, H. H. Complex pattern formation by adhesion-controlled anisotropic wrinkling. Langmuir 24, 6897-6902 (2008).

19. Breid, D. \& Crosby, A. J. Effect of stress state on wrinkle morphology. Soft Matter 7, 4490-4496 (2011)

20. Chen, Y. C. \& Crosby, A. J. Wrinkling of inhomogeneously strained thin polymer films. Soft Matter 9, 43-47 (2013).

21. Kim, P., Abkarian, M. \& Stone, H. A. Hierarchical folding of elastic membranes under biaxial compressive stress. Nat. Mater. 10,952-957 (2011).

22. Gao, G. X., Chen, X. \& Li, C. R. Self-assembled triangular and labyrinth buckling patterns of thin films on spherical substrates. Phys. Rev. Lett. 100, 036102 (2008).

23. Yin, J. et al. Stress-driven buckling patterns in spheroidal core/shell structures. Proc. Nat. Acad. Sci. USA 105, 19132-19135 (2008).

24. Chen, X. \& Yin, J. Buckling patterns of thin films on curved compliant substrates with applications to morphogenesis and three-dimensional micro-fabrication. Soft Matter 6, 5667-5680 (2010).

25. Li, B. et al. Surface wrinkling patterns on a core-shell soft sphere. Phys. Rev. Lett. 106, 234301(2011).

26. Cao, Y. P., Li, B. \& Feng, X. Q. Surface wrinkling and folding of core-shell soft cylinders. Soft Matter 8, 556-562 (2012).

27. Trindade, A. C. et al. Wrinkling labyrinth patterns on elastomeric janus particles. Macromolecules 44, 2220-2228 (2011).
28. Breid, D. \& Crosby, A. J. Curvature-controlled wrinkle morphologies. Soft Matter 9, 3624-3630 (2013).

29. Cai, S. et al. Periodic patterns and energy states of buckled films on compliant substrates. J. Mech. Phys. Solids 59, 1094-1114 (2011).

30. Zhao, T. \& Qiu, D. One-pot synthesis of highly folded microparticles by suspension polymerization. Langmuir 27, 12771-12774 (2011).

31. Liu, S. Q., Deng, R. H., Li, W. K. \& Zhu, J. T. Polymer microparticles with controllable surface textures generated through interfacial instabilities of emulsion droplets. Adv. Funct. Mater. 22, 1692-1697 (2012).

32. Yu, H. F. et al. Wrinkled liquid-crystalline microparticle-enhanced photoresponse of PDLC-like films by coupling with mechanical stretching. Small 7, 3039-3045 (2011).

33. Shih, T. K. et al. Topographic control on silicone surface using chemical oxidization method. Appl. Surf. Sci. 253, 9381-9386 (2007).

34. Watanabe, M. \& Mizukami, K. Well-ordered wrinkling patterns on chemically oxidized poly(dimethylsiloxane) surfaces. Macromolecules 45, 7128-7134 (2012)

35. Koh, A. et al. Flocculation and coalescence of oil-in-water poly(dimethylsiloxane) emulsions. J. Colloid Interface Sci. 227, 390-397 (2000).

36. Jiang, K. Q. et al. Microfluidic synthesis of monodisperse PDMS microbeads as discrete oxygen sensors. Soft Matter 8, 923-926 (2012)

37. Benedetto, F. D. et al. Rolling particle lithography by soft polymer microparticles Soft Matter 9, 2206-2211 (2013).

38. Hyun, D. C. \& Jeong, U. Substrate thickness: an effective control parameter for polymer thin film buckling on PDMS substrates. J. Appl. Polym. Sci. 112, 2683-2690 (2009).

39. Silicone Handbook [Ito, K. (ed.)] (Nikkan Kogyo Shinbun-sha, Tokyo, 1990).

\section{Acknowledgments}

Lu, CH acknowledges the financial support from NSFC (No. 21074090, 21374076), Tianjin Research Program of Application Foundation and Advanced Technology

(No.13JCYBJC17100). Cao YP acknowledges the financial support from NSFC (No. 11172155) and Tsinghua University (2012Z02103).

\section{Author contributions}

C.H.L. initiated the project, conceived and supervised the experiments, analyzed the data and wrote the paper. J.Y., X.H. prepared and characterized the samples. Y.P.C. provided assistance with the analysis of the data and the revision of the paper.

\section{Additional information}

Supplementary information accompanies this paper at http://www.nature.com/ scientificreports

Competing financial interests: The authors declare no competing financial interests

How to cite this article: Yin, J., Han, X., Cao, Y. P. \& Lu, C. H. Surface Wrinkling on Polydimethylsiloxane Microspheres via Wet Surface Chemical Oxidation. Sci. Rep. 4, 5710; DOI:10.1038/srep05710 (2014)

This work is licensed under a Creative Commons Attribution-NonCommercialNoDerivs 4.0 International License. The images or other third party material in this article are included in the article's Creative Commons license, unless indicated otherwise in the credit line; if the material is not included under the Creative Commons license, users will need to obtain permission from the license holder in order to reproduce the material. To view a copy of this license, visit http:// creativecommons.org/licenses/by-nc-nd/4.0/ 